\section{Clase, conciencia y cultura obrera en la Huelga de la Construcción (1935-1936)}

\author{
(4) Joaquina de Donato Lozano \\ Universidad de Buenos Aires. Facultad de Filosofía y Letras. Instituto \\ Interdisciplinario de Estudios e Investigaciones de América Latina, Argentina / \\ joaquina.dedonato92@gmail.com
}

Fecha de recepción: 1/9/2018. Fecha de aceptación: 7/2/2019.

\begin{abstract}
Resumen
El 23 de octubre de 1935, el Sindicato de Obreros Albañiles, Cemento Armado y Anexos de la Capital Federal dio comienzo a una de las huelgas de mayor envergadura de la década de 1930. Durante 94 días, más de 60.000 obreros, organizados por el Partido Comunista y con el apoyo del grupo Spartacus, sostuvieron una ardua confrontación en exigencia de mejores condiciones salariales y reconocimiento del sindicato. La intransigencia de la patronal se combinó con la poca flexibilidad del Departamento Nacional de Trabajo y la sólida resistencia de los obreros para desembocar en la Huelga General de los días 7 y 8 de enero de 1936. El presente trabajo se propone analizar la huelga descrita como manifestación de la dinámica y características del movimiento obrero argentino en un período particular de su formación (1930-1943) y la relación que entabló el proletariado industrial con el Partido Comunista en los años previos a la llegada del peronismo.
\end{abstract}

\section{Class, consciousness and working class culture in the Construction Worker's Strike of 1935-1936}

\begin{abstract}
On October 23rd, 1935, the Trade Union of Bricklayers, Armed Concrete and Annexes of Capital Federal began one of the largest strikes of the 1930s. For 94 days, more than 60,000 workers, organized by the Communist Party and with the support of the anarchist group Spartacus, sustained an arduous confrontation demanding better salary conditions and recognition of their union. The intransigence of the employers together with the little flexibility of the National Department of Labor and the solid resistance of the workers, led to the General Strike on January 7th and 8th, 1936. The present paper aims to analyze the strike described above as a manifestation of the dynamics and characteristics of the Argentine labor movement in a particular period of its formation (1930-1943) and the relationship that the industrial proletariat established with the Communist Party in the years prior to the arrival of Peronism.
\end{abstract}

\section{Palabras clave}

Partido Comunista Huelga

Obreros de la construcción

\section{Key words}

Communist Party Strike

Trade Union of Bricklayers Armed Concrete and Annexes 


\section{Introducción}

Para la época en la que se enmarca la huelga de la construcción, la integración que había logrado la Argentina dentro del mercado mundial ocasionó, inevitablemente, que al producirse la crisis desencadenada por la caída de la Bolsa en Wall Street (1929), su economía se viera fuertemente afectada. La caída de los precios en Europa significó la disminución en el volumen y valor de las exportaciones, contrayendo los beneficios del sector agropecuario al cual el país debía su prosperidad (Rapaport, 2012: 206). Luego de un breve impasse en el que los sectores dominantes se resistieron a la aplicación de políticas económicas heterodoxas, se volvió inevitable la necesidad de que la Argentina se adecuara a las nuevas condiciones estructurales del comercio mundial. Comenzó así, con el gobierno de Agustín P. Justo, un período de innovaciones cuyo objetivo no fue tanto la modificación de la relevancia del sector agrícola en el campo económico sino la complementación del mismo con el sector industrial. Lógica que se debió, más que nada, a que los cambios no fueron producto de un proyecto preestablecido sino la continua adaptación a los vaivenes del panorama internacional (Torre, 2011:45).

Este nuevo proceso, caracterizado por la "industrialización por sustitución de importaciones", un Estado propulsor de medidas proteccionistas y la consecuente expansión del mercado interno, potenció un rápido crecimiento del sector industrial que comenzó a revertir los altos índices de desocupación producidos por la crisis. Sin embargo, la expansión se mantuvo inmune a modificar las condiciones salariales (de hecho la velocidad del desarrollo de la industria tuvo mucho que ver con la disponibilidad de mano de obra barata) o la legislación laboral.

En lo que se refiere a la situación de la clase obrera, la década del treinta asistió al crecimiento de un proletariado industrial nuevo, numeroso y concentrado, mayoritariamente semicalificado o descalificado, en condiciones de explotación extrema y de escasa representación tanto en el terreno político como en el sindical. Fue ahí donde se abrió la posibilidad de inserción de partidos de izquierda, sobre todo el comunista (Camarero, 2012: 64).

Cabe destacar que no siempre la historiografía ha prestado la debida atención a este vínculo. Solo a partir de los trabajos de Juan Carlos Torre (2011/1990), Hugo del Campo (1983), Miguel Murmis y Juan Carlos Portantiero (2004/1971) entre fines de los sesenta y principios de la década siguiente, la Academia comenzó un reconocimiento de la inserción del comunismo en el movimiento obrero industrial, si bien es verdad que la temática no fue puntualmente abordada por estos autores. Más específicamente pueden mencionarse la investigación inconclusa de Celia Durruty (1969) y los trabajos de José Aricó (1979) a fines de los años setenta. Sin embargo, distinguimos un salto cualitativo en la historiografía abocada a la relación entre partidos de izquierda y clase obrera a partir de la década del 2000 con los pormenorizados trabajos de Nicolás Iñigo Carrera (2000), Hernán Camarero (2007), Diego Ceruso (2015) y Mariana Mastrángelo (2011). Si bien conforman puntos de vista muy disímiles, la calidad y extensión de sus investigaciones resultan los referentes más significativos que serán tenidos en cuenta en el presente trabajo pues implícitamente todos ellos tratan de dar respuesta a la misma pregunta: ¿cómo fue la relación entre partidos de izquierda y movimiento obrero en los años previos al advenimiento del peronismo y dónde pueden hallarse las causas de la exitosa inserción de los mismos entre el proletariado fabril?

A fin de llevar adelante un intento por colaborar a los mismos objetivos, la presente investigación vuelve necesaria la previa definición de los conceptos bajo los cuales se desarrollará nuestro marco interpretativo. Esto además es necesario ya que nuestra postura contrasta marcadamente con los lineamientos metodológicos de quien más minuciosa y exhaustivamente ha analizado la Huelga de la Construcción: Nicolás Iñigo Carrera. 
La siguiente definición de clase obrera se deriva del marxismo británico y de la propuesta por un enfoque "desde abajo" para entender los acontecimientos de 19351936. Es así que entendemos que las clases sociales se configuran como tales a partir de una interrelación tripartita de factores objetivos y subjetivos. En primer lugar, la posición estructural de los sujetos dentro de un modo de producción determinado en un momento determinado, el cual delimita uno de los principales ejes organizadores de la vida del obrero: la explotación. En segundo lugar, distinguimos la confrontación con otras clases sociales en tanto entendemos, como lo sostuvo E.P. Thompson, que las clases no existen como entidades separadas, que miran en derredor, encuentran una clase enemiga y empiezan luego a luchar. Por el contrario, las gentes se encuentran en una sociedad estructurada en un momento determinado [...], experimentan la explotación [...], identifican puntos de interés antagónicos, comienzan a luchar por estas cuestiones y en el proceso de lucha se descubren como clase, y llegan a conocer este descubrimiento como conciencia de clase (Thompson, 1989: 37).

Así, el antagonismo de clase, además de ser previo a la conciencia, depende en gran medida de cómo los sujetos involucrados perciben su experiencia. Es decir, esa percepción es igual de relevante que la experiencia de la explotación en sí misma pues sólo cuando una clase llega a vivir su situación como injusta puede haber lugar para una acción transformadora. A su vez (y como tercer factor), la forma en la que los sujetos perciben su experiencia se encuentra anclado en una serie de significaciones culturales, entendiendo "cultura" como un "proceso ordinario" tal cual fue planteado por R. Williams (2001). En el caso del movimiento obrero argentino, encontramos que su cultura se rige por dos fundamentos básicos e interrelacionados: un alto grado de solidaridad intra-clase (que queda por encima de cualquier adherencia ideológica y es expresión de su cohesión como clase) y la percepción de un "nosotros" frente a un "otros". Ambos factores, condicionados por la experiencia de la explotación y la posición estructural de la clase. ${ }^{1}$

Adherir al descripto marco teórico-metodológico es lo que nos llevará a contrastar tan marcadamente con la interpretación de Nicolás Iñigo Carrera ya que su planteo parte de una definición de clase social que excluye a la cultura como elemento constitutivos de la clase, considerándolo meramente un "término vago e impreciso (...) que pierde utilidad si se pretende conocer alineamientos y enfrentamientos sociales, en los que las formas de la conciencia se manifiestan abiertamente en la meta que se propone, el enemigo que se constituye, los aliados que establece y los elementos que utiliza una fuerza en lucha" (Iñigo Carrera, 2000: 105). Partiendo de una lógica que presupone que sólo en el enfrentamiento con otras clases los sujetos se constituyen como tales, Iñigo Carrera propone que en esas luchas puede descubrirse la existencia de una "estrategia" que ordena los conflictos, la cual, a su vez, guarda relación con el grado de desarrollo de la conciencia de una clase en un momento determinado. Esta "estrategia" puede no ser consciente para la voluntad individual, sin embargo lo es para la clase entendida como sujeto colectivo bajo los condicionamientos de un proceso histórico determinado (Iñigo Carrera, 2000: 16-21). En otras palabras, "los enfrentamientos se ordenan siguiendo una estrategia, no importa el grado de desarrollo de la conciencia que se tenga de ella" (Iñigo Carrera, 2000: 24). Así el resultado de un conflicto se dirime en base a fuerzas que trabajan "inconscientemente" en relación a una "necesidad histórica", como fue bien señalado por Paula Varela (Varela e Iñigo Carrera, 2015: 160).

Por el contrario, queriendo alejarnos de interpretaciones que parecerían proponerse disociar a la conciencia de los sujetos y de los procesos sociales, nosotros entendemos que la conciencia es la síntesis inconclusa de cómo se percibe una experiencia que está anclada en la posición estructural de la clase en el proceso productivo y mediada por
1. Todo el descrito marco teórico-metodológico puede hallarse en mayor detalle en Pisani (2016), así como en otras publicaciones de la autora. 
una serie de significaciones culturales propias de un proceso histórico determinado. Así, experiencia, cultura y conciencia son elementos que se interrelacionan dinámica y continuamente. Y percibirlos es necesario para interpretar las acciones de los sujetos que se pretende conocer sin recaer en explicaciones de corte teleológico.

Lo que sigue a continuación es un intento por entender el proceso huelguístico de 1935-1936 "desde abajo", tratando de desentrañar las percepciones y motivaciones de los sujetos envueltos sin atribuirle a sus acciones una necesidad histórica o una "estrategia". Más bien el objetivo radica en lograr ver en la dinámica de esos 96 días de lucha la manifestación de las características del movimiento obrero argentino en un momento particular de su desarrollo y las vías a través de las cuales partidos de izquierda como el Comunista lograron insertarse dentro del proletariado industrial. Para tal fin no solo nos valdremos de la historiografía previamente mencionada sino que además recurriremos a entrevistas orales y escritas a militantes del período, a las memorias de dirigentes comunistas y anarquistas, a los artículos de la prensa de izquierda la cual siempre reservaba un espacio para la publicación de cartas escritas por obreros, y a los comunicados y panfletos provenientes del terreno sindical.

\section{Y los obreros cuando regresan a sus guaridas, en voz baja, cortante y grave, se pasan la orden: ¡No aflojar! ¡Firmes!”; Huelga de la Construcción (1935-1936)}

Corría el año 1929 cuando la Sociedad de Resistencia de Obreros Albañiles y Anexos (enrolada en la FORA) llamó al inicio de una huelga en reclamo por la abolición del trabajo a destajo, elevación de los salarios mínimos, descanso dominical y reconocimiento del sindicato (por nombrar algunos de las demandas planteadas en el pliego de condiciones entregado a la patronal). La huelga duró casi un mes, llevándose el saldo de un obrero muerto y varios heridos, y finalizó sin que ninguna de las demandas fuesen acogidas por la patronal. El balance hecho por el PC fue severamente duro con los anarquistas, acusándolos de no haber preparado a los obreros para el enfrentamiento (ni con la patronal ni con la policía) y de no haber sabido organizar comités de huelga ni apelado a la solidaridad para sostener económicamente a los huelguistas mientras duraba el conflicto (Camarero, 2007: 138). Seis años después, veremos al PC a la cabeza de una nueva huelga de la construcción poniendo en juego todos los elementos que habían criticado a los ácratas por no utilizar. Así, el plazo transcurrido entre ambas huelgas no solo nos anticipa modalidades de lucha que comenzaron a tener éxito a lo largo de los años 30 sino que además da cuenta de los cambios en la inserción del PC dentro del proletariado industrial. Y de cómo se trató de un proceso lento pero continuo que puede rastrearse a los primeros años de la década de 1920.

Según Hernán Camarero, esta mutación debe comprenderse a partir del cambio de orientación vivido por el PC a partir de 1925. Según el autor, la implantación de la estrategia de la "proletarización" y "bolchevización" del partido y la reorganización de los afiliados en torno al establecimiento de una organización celular le posibilitaron al PC transformarse en un verdadero partido proletario y cosechar adherencias en ese ámbito, sobre todo el fabril (Camarero, 2007: 2-3). Ambas modificaciones, a su vez, se adaptaron bien a los cambios políticos y económicos ocurridos desde fines de la década del 20. Así, el cambio en la propuesta política se combinó con una coyuntura determinada, convirtiendo al PC en un partido proletario articulado en torno a una identidad y una cultura obrera (Camarero, 2007: 350). Si bien coincidimos con los lineamientos generales esbozados, consideramos necesario darle mayor relevancia a las características de los colectivos obreros interpelados por el PC pues, como sostiene Juan Carlos Torre: "las ideologías nunca interpelan a un público inerte sino que los individuos concretos ya están culturalmente articulados por el complejo de actitudes, 
preferencias morales y tradiciones que es construido e incesantemente renovado a partir de sus condiciones de existencia" (Torre, 1990: 218). Es decir, el PC bien pudo haberse propuesto "conquistar" a la clase obrera y elaboró toda una serie de medidas para llevar adelante aquel cometido pero igual de relevante fueron las características de la cultura obrera de esa época que posibilitaron que se produjera una coincidencia entre ambos. Rescatamos, en este sentido, un comentario del dirigente comunista Domingo Varone:

\begin{abstract}
Sobre estas masas oprimidas convergían otras corrientes ideológicas, pero eran adoptadas como propias aquellas que reflejaban más directamente, más fácilmente, ese estado de insurgencia. El discernimiento ideológico vino más tarde, con la experiencia, a medida que se asimilaba aquella ideología que además de expresar los sentimientos de clase de los trabajadores, puso a la masa ante la evidencia de que su inmensa fuerza necesitaba una organización que le diera unidad (Varone, 1989: 29).
\end{abstract}

Siendo así, parecería que la incorporación al partido no viene dada por una adhesión a ciertos lineamientos ideológicos sino más bien por la coincidencia de elementos de esos lineamientos con "sentimientos de clase" o, utilizando los términos de R. Williams, con una "estructura de sentimiento" (1965: 57-60). En esa coincidencia, además, las características de los dirigentes son de gran importancia (Pozzi, 2015: 23). "El trabajador sencillo, con su buen olfato de clase - escribe Rufino Gomez ${ }^{2}$ en sus memorias - se guía por las actitudes de los hombres más capaces, los mejores en el trabajo, los de conducta más recta" (Gómez, 1973: 27). De hecho muchas veces las publicaciones del PC buscaban narrar acontecimientos (por lo general altercados con la policía) donde sus dirigentes hubiesen exhibido valores como la abnegación, coraje e intransigencia a fin de reforzar la legitimidad sobre su persona. En las diversas publicaciones que pudo editar el PC en la época, pueden rastrearse los componentes de la coincidencia entre cultura obrera y el partido. En estos periódicos, como señala Camarero, aparecen una serie de valores y nociones - como la valentía, la honradez, la dignidad, la humillación que se sufre del poderoso, el ser portador de justicia- que operan a manera de construcciones imaginarias que conforman el "mundo de los obreros" (Camarero, 2007: 55). En este sentido son muy interesantes las secciones donde se publican cartas anónimas de obreros que escriben para denunciar las excesivas condiciones de explotación, las pobres condiciones de trabajo y las injusticias perpetradas tanto por la patronal y los capataces como por la policía. En estas cartas anónimas siempre hay dos temas que se reiteran: la denuncia de la explotación y el llamado a la necesidad de organizarse para combatirla (por lo general no asociándolo a la necesidad por afiliarse a un partido político). Por tal razón, llama la atención lo escrito por un obrero del frigorífico Anglo: "Esa misma explotación nos está haciendo comunistas a todos los obreros del Anglo. ¿Qué otro camino nos queda sino seguir las líneas que nos señalan los comunistas, o morimos de hambre?". Y firmó: "un obrero que se hace comunista". ${ }^{3}$ Así, podría sugerirse que la voluntad explícita por incorporarse al PC se relaciona más con la capacidad que éste exhibió para organizar a los obreros y defender sus reivindicaciones que por una adherencia a puntuales lineamientos ideológicos por parte de los militantes. Por supuesto, para los dirigentes del PC uno es consecuencia de lo otro, pero eso no significa que los obreros tuviesen que haberlo percibido en esos mismos términos.

A su vez, cabe señalar que el trabajador durante una experiencia de lucha (posibilitada por la organización propulsada por la ideología del partido junto con otra serie de factores), sí genera cambios en la conciencia de dicho obrero y en la forma en la que construye la percepción de su experiencia de vida. Y al mismo tiempo, el mismo PC se va construyendo durante ese proceso de lucha. Por ende la relación entre propuesta política (organizativa) y la politización (individual y colectiva) es sumamente compleja y dinámica.
2. Rufino Gómez fue un dirigente del PC, perteneciente a la Unión Obrera Provincial (Córdoba).

3. Diario "Bandera Roja". Año 1. N7. 30-08-1932. "Del frigorífico Anglo". Puede ser consultado en el Centro de Documentación e Investigación de la Cultura de Izquierdas. 
4. Tomado de entrevista citada en Iñigo Carrera (2000: 151).

5. Diario “Bandera Roja”. Año 1. N18. 18-04-1932 “Como se explotan a los obreros en la obra de San Juan y Pozos".
Así, para 1935, encontramos a un PC inserto con éxito en varios sectores del proletariado fabril, bajo un contexto económico y social que propició un aumento en los niveles de explotación de la clase obrera en general y de ese sector en particular, y un clima político con altos niveles de hostilidad hacia el fraudulento gobierno de turno. Sumado a eso, el revestimiento de la crisis y el aumento en la producción de manufacturas ayudado por las políticas de sustitución de importaciones, así como revirtió los altos índices de desocupación también inició un ciclo huelguístico a partir de 1934, ciclo en donde cabe enmarcar la huelga de la construcción llamada por el Sindicato de Albañiles, Cemento Armado y Anexos un 23 de octubre (Ceruso, 2015: 121).

Como mencionamos ya, la explotación como hecho en sí mismo no es causa explicativa suficiente de por qué la huelga se desencadenó, mucho menos de por qué tomó el rumbo y duración que alcanzó. Sin embargo, la denuncia de la misma sí nos ilumina acerca de "mucho odio contra la burguesía por la actitud de la patronal de la construcción". ${ }^{4} \mathrm{Al}$ mismo tiempo, también es verdad que las consecuencias de condiciones de explotación extrema, sobre todo la muerte, muchas veces actúan como desencadenante de un conflicto. En la huelga de la construcción, por ejemplo, tanto Iñigo Carrera como Celia Durruty recogen a partir de entrevistas que el clima para la huelga ya estaba gestado a partir del derrumbe de una obra en Republiquetas y Cabildo que produjo diez muertos y varios heridos. "La C.D del sindicato cree que no es posible que este hecho quede en silencio ya que la situación de la obra en que ha ocurrido este desgraciado accidente no es la única en que se trabaja en pésimas condiciones sino que en todas las obras la muerte nos acecha, por las condiciones en que debemos realizar el trabajo, con el peor material posible y andamiaje con maderas podridas" quedó escrito en un volante (Durruty, 1969: 79). También, en este sentido, son varias las cartas de obreros de la construcción publicadas en los diarios militantes La Internacional y Bandera Roja, durante esos años, que hacen referencia a lo mismo. "Un obrero de la obra" publicó en 1932, por ejemplo, "Compañeros que trabajan allí (en la obra) yo por medio de nuestro querido diario Bandera Roja os quiero incitar a organizarse para acabar con esta explotación y una vez organizados iremos a la lucha por nuestras reivindicaciones". 5

El 8 de septiembre, bajo asambleas convocadas por la FOSC, se decidió la entrega de un pliego de condiciones (que fue presentado el 22 de octubre) y el llamado a la huelga (con la consecuente creación de un Comité de Huelga) en caso de que éste no fuese atendido (Camarero, 2009).

El pliego estuvo compuesto por 17 cláusulas entre las cuales cabe destacar la exigencia por el reconocimiento del sindicato y delegados en las obras, el reclamo salarial, toda una serie de disposiciones en torno a fijar mejores condiciones de trabajo y salubridad, y la exigencia de pautas acerca de cómo debían estar construidos los andamios de las obras detallado minuciosamente en 13 puntos. Esto último es interesante por dos cuestiones. En primer lugar, este tipo de puntos permite acceder a la forma en la que la cultura obrera percibe al trabajo. Esto es, el trabajo es fuente de dignidad y se engrandece a aquel que sabe realizarlo (Mastrángelo, 2014: 33). Probablemente por eso en los periódicos siempre la patronal es "vaga" (sabrá de dinero pero no sabe de trabajo). En segundo lugar, si bien puede considerarse la construcción de los andamios como un ítem más relacionado a la búsqueda por preservar la salud del obrero durante las obras, también implícitamente está significando un cuestionamiento a la propiedad privada de los medios de producción. Es decir, fijar con qué materiales, con qué medidas y en qué circunstancias deben ser construidos los andamios está imponiendo límites a los ritmos del proceso productivo, indistintamente que en el pliego no esté redactado en esos términos.

El mismo 22 de octubre, las organizaciones patronales respondieron al pliego, solamente contestando a la demanda por el reclamo salarial e indicando la escala que 
estaban dispuestos a pagar. Tal cual lo acordado, el 8 de septiembre, los obreros de las principales empresas de la construcción abandonaron el sitio de trabajo y se dirigieron al Luna Park donde tuvo lugar una asamblea (Iñigo Carrera, 2000: 129).

A partir de ese momento comenzó a exhibirse todo el despliegue organizativo incentivado por el PC, el cual, como manifestó uno de los testimoniantes, fue "muy perfecto". Entre las varias acciones lanzadas a fin de lograr el mantenimiento de la huelga, se destacó la formación de Comités por barrios, la organización de comedores y mitines, y el armado diario de grupos de piquetes que salían a controlar la paralización de las obras. A su vez, y este es uno de los datos que protagonistas y testigos más destacaron del conflicto, se apeló a la solidaridad para mantener a los huelguistas. Como sostuvimos ya, es uno de los fundamentos básicos de la cultura obrera de la época y durante la huelga de la construcción tenemos la oportunidad de verlo manifestarse. Esta solidaridad se desprende del proceso productivo y se alimenta y retroalimenta en el antagonismo de clase fundado, no sobre cuestiones ideológicas, sino sobre determinantes clasistas, raciales y de género (Pozzi, 2011). Por ende, en todo momento encontramos que la solidaridad se halla por encima de cualquier tipo de adhesión política. Como sostuvo el dirigente obrero Miguel Contreras en sus memorias: "Esa es la democracia obrera y la aplicábamos" (Contreras, 1978: 43). O como contó un entrevistado acerca de los barrios que luego se vieron envueltos en la huelga: "En los barrios hay otra fraternidad, hay otra convivencia. Entonces, ahí se pasa un poco por encima de las ideologías. Es de persona a persona. De grupo a grupo" (Iñigo Carrera, 2000: 106). Esto no significa confundir solidaridad con armonía. El mismo testimonio señala que "Había mucho chusmerío. Se peleaban. Pero cuando hacía falta solidaridad respondían". Y también es pertinente recordar que durante el conflicto muchas veces la violencia de los huelguistas no fue dirigida a las empresas sino a aquellos trabajadores que no habían hecho abandono del sitio de trabajo (Durruty, 1969: 81).

Dicha solidaridad, a su vez, fue un terreno en el que el activismo femenino tuvo oportunidad de expresarse, el cual fue indispensable para que la huelga pudiese extenderse en el tiempo. Comedores populares, centros de asistencia médica y organizaciones de amas de casa de apoyo a la huelga fueron todos espacios "no productivos" (tener en cuenta que el ámbito de la construcción era un campo netamente masculino) que madres, esposas e hijas tuvieron oportunidad de interpelar (D’Antonio, 2000: 251).

Para Iñigo Carrera, la "estrategia" de la clase obrera en un determinado momento histórico la lleva a establecer alianzas con sectores no proletarios a fin de lo cual necesita plantear su interés de manera tal que pueda ser aceptado como propio por facciones sociales de otras clases (Iñigo Carrera, 2000: 21). Así el autor explica la generalización de la solidaridad durante la huelga (y sobre todo en la huelga general de los días 7 y 8). Sin embargo, como fue bien observado por Celia Durruty, la solidaridad solo se buscó dentro de los espacios de sociabilidad obrera, los cuales, por lo general, estaban circunscriptos al ámbito barrial. Es por eso que los testimonios, por lo general, registraron la colaboración del almacenero, el médico del barrio, el panadero, el colectivero, el carnicero, etc. Al mismo tiempo, nunca puede dejar de recordarse que la solidaridad brindada por facciones ajenas a la clase obrera, así como el apoyo de otros partidos políticos o federaciones sindicales, también estuvieron influenciados por un contexto económico y político particular.

Siendo así, el éxito del PC puede ser entendido a partir de su destreza por saber canalizar una solidaridad preexistente en organizaciones y acciones concretas destinadas a asegurar el mantenimiento en el tiempo de la huelga. De hecho, quizás podría pensarse que la solidaridad funcionó como un nexo entre el partido y la "estructura de sentimiento" de la clase obrera en ese momento. Nuevamente aquí entra a jugar la compleja dinámica entre propuesta política organizativa y politización. Como señaló 
6. Dirigente del PC y Secretario General del Sindicato de Obreros Albañiles, luego de la sección Capital de la Federación Nacional de Obreros de la Construcción, fundada como consecuencia de la huelga de 1936.

7. Dirigente del PC, Secretario General del Sindicato de Obreros Albañiles, Cemento Armado y Anexos de la Capital Federal y en la década de cuarenta, vicepresidente del Comité Central Confederal de la CGT.
Mariana Mastrángelo, la lucha de clases no es solo un componente del discurso marxista sino que atraviesa la vida de los sujetos y las determina al punto de que su respuesta para hacer frente a la explotación y a la injusticia es el ingreso a la política (Mastrángelo, 2009: 399). En paralelo, es más que constatable cómo la reputación y solidez del PC se fueron fortaleciendo a lo largo del conflicto, permitiendo que su proceso de organización experimentara significativos avances (Camarero, 2009). Rubens Íscaro ${ }^{6}$ destacó que la experiencia de la huelga tonificó "en medida considerable" al movimiento sindical en su conjunto (Íscaro, 1958: 152). Por ende, en la lucha, tanto el partido como los proletarios experimentaron un salto de calidad que expandió los límites de sus posibilidades y permitió la perduración de la huelga en el tiempo. Como dejó constancia un participante: "El elemento organizativo fue tan importante que fue el que permitió darle esa envergadura a la huelga general del $7 \mathrm{y}$ 8 de enero. La repercusión que tiene esa huelga no surge espontáneamente. Surge de esta trama que se ha ido construyendo durante dos meses de solidaridad, con una actividad muy esclarecedora de los obreros" (Iñigo Carrera, 2000: 139).

Por otro lado, a medida que el conflicto se prolongó, comenzó a aparecer un tercer actor: el Estado. La aceptación de los huelguistas por permitir la intervención del DNT como mediador es uno de los elementos que utiliza Iñigo Carrera para plantear cómo "la estrategia de la clase obrera" es el ingreso al sistema institucional. Sin embargo, basta con ver la narración de la reunión hecha por el Secretario General del sindicato, Pedro Chiarante, ${ }^{7}$ para percibir que en realidad se trata de algo mucho más complejo:

(...)El mismo ministro que había tratado de liquidar por la fuerza y por el hambre nuestra lucha quien nos llama a su lujoso despacho y en tono amable afirma que el gobierno está profundamente conmovido por los hechos que son de conocimiento público (...) ¡Conmovedor! Los compañeros comentaron luego que salieron deslumbrados ante tamaña muestra de habilidad, hipocresía y cinismo del ministro justicia. Decían que en algún momento pensaron que no podrían disimular la indignación (...) pero recordaron que en la mesa de conversaciones las simpatías personales no deben contar (...) aunque se efectúe entre dos enemigos que pocos instantes antes se ocupaban de destriparse uno a otro con toda prolijidad (Chiarante, 1976: 196).

Como sostuvo Hugo del Campo (1983: 63), la clase obrera solía buscar el apoyo del poder político a fin de concretar sus reivindicaciones, pero que no se reniegue de la participación del Estado no es lo mismo que sostener que se desease la incorporación al sistema. No debe olvidarse que la ausencia de una legislación laboral de alcance general termina por volverlo necesario (Torre, 2011: 66). Por otro lado, que a lo largo del conflicto no haya habido una asociación por parte de los sectores proletarios entre los hechos y la búsqueda por la superación al sistema tampoco es lo mismo que decir que los obreros "querían" incorporarse al sistema, como tampoco es lo mismo que decir que si la posibilidad se hubiese presentado, ellos la habrían desdeñado. Negociar no implica compartir la ideología ni aceptar el orden social vigente sino que, más bien, está relacionado con la búsqueda por alcanzar un trato directo con funcionarios claves del aparato estatal a fin de poderles plantear sus reivindicaciones (Torre, 2011: 68). Así, no es casualidad que un par de años luego de finalizada la huelga, Rubens Íscaro haya sostenido que los organismos gubernamentales fueron utilizados a fin de lograr el éxito de la huelga (Iñigo Carrera, 2000: 239).

Sin embargo, la intransigencia de los sectores patronales y la austeridad de las propuestas volvió poco eficaz la intervención del DNT. De todos modos, es cierto que a partir de su intervención logró quitarse de la mesa de discusión la demanda por el reconocimiento del sindicato, acotando la negociación a una lucha en torno a reivindicaciones salariales. 
En paralelo al comienzo de negociaciones, el 7 de diciembre de 1935 se formó el Comité de Defensa y Solidaridad con los Obreros de la Construcción el cual fue el responsable de dar llamado a la huelga general de los días 7 y 8 de enero de 1936.

\section{"Su triunfo significa el triunfo de todos. Su derrota es nuestra ruina"; 7 y 8 de enero de 1936}

Desde diciembre de 1935, mientras tomaban lugar las negociaciones con la patronal y el DNT, y mientras se asiste a formación del Comité de Defensa y Solidaridad, son constatables una serie de enfrentamientos callejeros entre la policía y los huelguistas lo cual sumado a los casi dos meses de mantenimiento de la huelga ayuda a explicar que se abandonara la pretensión por el reconocimiento del sindicato y se lograse centrar el conflicto en torno a cuestiones salariales. Sin embargo, la intransigencia de los sectores patronales frustró todo intento de acuerdo mediado por el DNT con lo cual la huelga siguió su curso. Finalmente, a principios de enero de 1936, el Comité de Defensa y Solidaridad encabezado por Mateo Fossa, ${ }^{8}$ llamó a la huelga general para el día 7 de enero alegando que "El conflicto de los obreros de la Construcción no es un hecho aislado y esporádico. Nos afecta a todos por igual y es vitalmente necesario para el movimiento sindical prestar la más amplia y generosa ayuda al mismo".?

Desde el 6 de enero, a partir de la difusión a través de los barrios, comenzaron a propagarse las actividades que tuvieron lugar. Y la policía aprovechó estos anuncios para preparar sus fuerzas a fin de dispersar a los huelguistas.

La mañana del 7, núcleos de obreros se formaron en las puertas de las fábricas "comunicando a los trabajadores que llegaba la resolución de la huelga general: "inadie entra al trabajo!" (Íscaro, 1959: 155). Alrededor de las 7 de la mañana tuvo lugar el primer tiroteo cobrándose un saldo de 19 muertos y heridos (policías y obreros) (Iñigo Carrera, 2000: 177). Lo que siguió fue un vertiginoso número de acontecimientos entre manifestaciones, mitines, cortes de calle, enfrentamientos con la policía, quema de colectivos a fin de impedir la circulación, arrestos y otra serie de eventos, donde se mezcló la actividad espontánea de los huelguistas con acciones planificadas desde los cuadros del PC. Todo esto llevó al Comité de Defensa y Solidaridad a extender el paro otras $24 \mathrm{hs}$.

Esto fue posible, como fuimos viendo en el apartado anterior, gracias a altos niveles de organización y solidaridad, que se extendieron a sectores no proletarios aunque, como ya sostuvimos, se mantenían dentro de los mismos ámbitos de sociabilidad. Según Iñigo Carrera, en estos dos días de enfrentamientos asistimos a un "combate de masas" donde por un lado se encontraban las fuerzas "proletarias y populares" y por el otro la patronal, el gobierno "y potencialmente el Estado mismo" (Iñigo Carrera, 2000: 227). Así, un conflicto que había acabado centrado en reivindicaciones económicas devino, de pronto y sin buscarlo, en una lucha política lo cual revela "la otra estrategia de la clase obrera" que es la momentánea búsqueda por la superación del orden social vigente. Sin embargo, alrededor de dos semanas después, el conflicto finalizó por medio de una negociación con la patronal. ¿Cómo explica el autor este cambio? "El hecho de que finalmente se imponga la estrategia de incorporarse al sistema institucional se corresponde con la tendencia dominante en un momento histórico" (Iñigo Carrera, 2000: 289).

En primer lugar, quizás pueda aceptarse que la huelga general devino en una "huelga de masas política" (aclarando que no fue convocada en esos términos ni tampoco excedió una reacción espontánea de descontento social) pero mucho más difícil es sostener que
8. Militante del PC, Fossa había protagonizado la gran huelga de la madera que se desarrolló entre mayo y julio de 1935. El reconocimiento por su rol en el gremio de la madera y durante la huelga, impulsó su participación como Secretario del Comité de Defensa y Solidaridad durante la huelga de la construcción.

9. Tomado de "Carpeta Huelga 1936" compilado por el CEDINCI. 
10. Mateo Fossa “Características, experiencias y resultados de la huelga de la construcción" consultado en https://www. laizquierdadiario.com/Mateo-Fossadirigente-obrero el $14 / 28 / 2018$. durante el conflicto se planteó una superación al sistema vigente. En ningún momento, ni siquiera cuando el Comité de Defensa y Solidaridad emitió un nuevo pliego en respuesta a los encarcelamientos de varios dirigentes sindicales, el reclamo obrero excedió la lucha por sus reivindicaciones. Es más, en ese mismo pliego se pidió al gobierno "que obligue a la patronal a reconocer las justas aspiraciones de los obreros de la construcción". En otras palabras, en ningún momento hay un viraje en las propuestas trazadas ni por los huelguistas ni por sus dirigentes. Los altos niveles de participación popular que alcanzaron los días 7 y 8 correspondería más bien explicarlos a partir de una extensión de la solidaridad sumada a "la sorda ira acumulada durante largos días de huelga" (Íscaro, 1958: 155). A lo cual, añádase también, un contexto político económico particular de altos índices de rechazo al fraudulento gobierno de turno.

Por otro lado, lo que más llama la atención de la huelga son los altos niveles de cohesión de la clase obrera como clase y de conciencia social. Como narra Fossa, haciendo un balance de la huelga en el diario de los albañiles El Andamio:

Los obreros albañiles que fueron los iniciadores de la huelga y que la mantuvieron solos por espacio de un mes y medio, trazaron las bases del plan de lucha. Ampliándose horizontalmente, la huelga de los albañiles consolidóse, con el aporte de nuevas fuerzas, pertenecientes a la F.O.S.C. Extendido el conflicto a todas las ramas de la industria, una masa de cerca de 60 mil obreros entró a luchar por sus reivindicaciones. Más adelante creóse el Comité de Defensa y Solidaridad, el cual, a fin de defender las demandas presentadas por los obreros de la construcción, hizo intervenir en la huelga general del 7 y del 8 de enero último, a toda la clase trabajadora de la capital y pueblos vecinos. ${ }^{10}$

El altísimo nivel de cohesión de los obreros como clase puede llevarnos a cuestionar si el comentario de Iñigo Carrera "solo puede haber dos formas de conciencia: socialista o burguesa" (Iñigo Carrera, 2000: 20) no recae en una excesiva simplificación que desdibuja la complejidad del problema y asigna roles y categorías a sujetos cuya experiencia corre por carriles muy alejados de tales teorizaciones. Si tenemos una huelga cuya duración y solidez es manifestación de un alto grado de desarrollo de la conciencia de la clase a su vez que esa conciencia no se propone la superación al sistema, quizás lo que esto nos está diciendo es que la conciencia se mueve contradictoria y dialécticamente y que su dinamismo es producto, como sostuvimos antes, de la interrelación de factores objetivos y subjetivos que conforman a un conjunto de trabajadores como "clase" (Pozzi, 2009: 94-95). Se vuelve así factible que una conciencia fuerte no tenga por qué devenir en socialista o que si bien no critique abiertamente el sistema capitalista al reivindicar su lugar como clase y al trabajo como fuente de dignidad, termine implícitamente cuestionando los pilares sobre los cuales se funda dicho modo de producción.

Dicha problematización respecto de la conciencia de clase como contradictoria, no lineal e inconclusa es también lo que nos lleva a sugerir una interpretación alternativa acerca de las causas del fin de la huelga que no pasen por sostener que la tendencia histórica dominante fue hacer que la "estrategia de la clase obrera" fuese su adhesión al sistema institucional. Para tal fin rescatamos un comentario que hace Domingo Varone en sus memorias: “(...)El luchador que desprecia a su propia vida, por muy heroico que aparezca, desprecia la propia causa por la que dice luchar" (Varone, 1989: 113). El comentario nos sugiere la existencia de una "dimensión práctica de la conciencia" (Pisani, 2016: 26) y nos advierte que debemos abstenernos de trazar una línea directa entre la percepción que un sujeto dentro de una clase hace de su experiencia y la realización de acciones concretas que coincidan con tales percepciones. En otras palabras, nos recuerda que entre lo que los sujetos "hacen" y lo que los sujetos "piensan" no hay una correlación directa sino que la misma depende de una variedad de factores en un contexto determinado. Siendo así, puede pensarse que la negociación con la patronal que puso fin a la huelga fue producto del interés de la 
clase obrera por ser parte del sistema institucional o bien puede pensarse que una huelga no puede perdurar infinitamente en el tiempo por mucha organización y solidaridad que haya en ella. Llegado un punto, la perduración de la huelga implica una amenaza para la reproducción material de los huelguistas causando que mantenerla termine por volverse en contra de los principios (sean meramente reivindicativos o ideológicos) sobre los cuales la misma se sostuvo.

Por otro lado, es común que se refiera al fin de la huelga como una "victoria parcial" porque se logró el reconocimiento salarial aunque no el reconocimiento del sindicato. Sin embargo, es bastante difícil decir que para los huelguistas (o incluso para el PC) la misma fuera vivenciada en esos términos. Después de todo, sostuvieron durante 96 días un conflicto con una patronal que todos caracterizaban por su intransigencia, obligándola a modificar las escalas salariales que estaban dispuestas a pagar, la reducción de la jornada laboral a 8 horas y la readmisión de los despedidos (Ceruso, 2015: 127). Y en lo que se refiere al no reconocimiento del sindicato, cabría alejar la renuncia a tal cláusula como la renuncia de las pretensiones obreras a tener su espacio de representación. De hecho, el impulso otorgado por el conflicto llevó a la conformación de la primera federación nacional de la industria a mediados de 1936: la FONC. Por ende, la finalización de la huelga, más que dar cuenta de una estrategia subyacente, nos remite a necesidades concretas de los huelguistas en un contexto determinado y cómo las mismas no necesariamente implican una redefinición de las percepciones obreras respecto de un "nosotros" y un "ellos".

\section{Conclusión}

En el libro Tiempos de insurgencia, se menciona cómo los momentos insurreccionales llevados adelante por las clases subalternas habitan una temporalidad radicalmente diferente a aquella implícita en los relatos dominantes (sean liberales o de izquierda) y que para acceder a ella se vuelve preciso romper con toda interpretación que entienda los procesos históricos (y su relevancia) a partir de la interrelación constante de causas y consecuencias, y situarse en cambio en el "tiempo real de la experiencia" (Adamovsky et al, 2006: 25). A lo largo del presente trabajo, buscamos seguir las huellas de tales lineamientos. Nuestra propuesta radicó en el intento de acceder a la larga huelga de los obreros de la construcción lo más cercano posible a como fue vivenciada por sus protagonistas, bajo qué necesidades, percepciones y expectativas, y cómo las mismas se interrelacionaron en un determinado y concreto momento histórico, propulsándose hacia un fin: el triunfo de la huelga y por ende del derecho de todo trabajador a un salario digno, una jornada laboral y condiciones de salubridad justas y a organizarse y nombrar representantes.

Siendo así, es nuestro entender que analizando la huelga en esos términos, la misma puede ser vista como manifestación de las características particulares de la clase obrera argentina en un momento particular de su desarrollo, lo cual implica que nos permite acceder a la cultura de los trabajadores en ella involucrados y al desarrollo de formas de conciencia (y cómo ésta se va complejizando y fortaleciendo durante el conflicto). Por otro lado, la huelga también evidencia las formas y calidad de los vínculos tendidos entre trabajadores y partidos de izquierda, como el PC, en la década del treinta. En este sentido, lo que podría evidenciar el conflicto es un alto nivel de coincidencia entre propuestas y expectativas de los obreros y aquellas de los dirigentes comunistas en un momento histórico determinado. Y cómo la perduración en el tiempo de dicha coincidencia puede producir cambios en la conciencia de los actores envueltos dando por resultado formas cada vez más elaboradas de organización y lucha, las cuales se vuelven capaces de trascender la experiencia concreta de la huelga y de los individuos en ella involucrados. 


\section{Q Bibliografía}

》Adamovsky, E. et al (2006). Tiempo de Insurgencia: experiencias comunistas en la revolución rusa. Buenos Aires.

" Aricó, J. (1979). “Los comunistas en los años treinta”. Buenos Aires: Controversia 2-3: v-vii.

"Camarero, H. (2007). A la conquista de la clase obrera. Los comunistas y el mundo del trabajo en la Argentina (1920-1935). Buenos Aires: Siglo XXI.

"Camarero, H. (2009). "Un sindicato comunista antes del advenimiento del peronismo: el caso de la Federación Obrera Nacional de la Construcción (FONC)". Actas de las XII Jornadas Interescuelas/Departamentos de Historia.

»Camarero, H. (2012). “Ascenso y ocaso del Partido Comunista en el movimiento obrero argentino: crítica historiográfica y argumentaciones conceptuales”. Archivos de historia del movimiento obrero y la izquierda, 1 (1), 57-79.

»Ceruso, D. (2015). La izquierda en la fábrica. La militancia obrera industrial en el lugar de trabajo, 1916-1943. Buenos Aires: Imago Mundi.

»Chiarante, P. (1976). Ejemplo de dirigente obrero clasista, Buenos Aires: Fundamentos.

»Contreras, M. (1978). Memorias. Buenos Aires: Ediciones Testimonios.

"D’Antonio, D. (2000). "Representaciones de género en la huelga de la construcción. Buenos Aires, 1935-1936"; en Gil Lozano, F. Pita, V; Ini, M (comps.). Historia de las mujeres en la Argentina, Tomo II. Buenos Aires: Taurus, pp. 245265.

»Del Campo, H. (1983). Sindicalismo y Peronismo. Los comienzos de un vínculo perdurable. Buenos Aires: CLACSO.

»Durruty, C. (1969). Clase obrera y peronismo. Córdoba: Ediciones Pasado y Presente.

"Gómez, R. (1973). La gran huelga petrolera de Comodoro Rivadavia (1931-1932) en el recuerdo del militante obrero y comunista Rufino Gómez. Buenos Aires: Ediciones Centro de Estudios.

"Iñigo Carrera, N. (2000). La estrategia de la clase obrera 1936. Buenos Aires: La Rosa Blindada.

» Iñigo Carrera, N. y Varela, P. (2015). "Diálogo sobre el concepto de 'estrategia' de la clase obrera". Archivos de historia del movimiento obrero y la izquierda, 3(6), $155-175$.

" Íscaro, R. (1958). Origen y desarrollo del movimiento sindical argentino. Buenos Aires: Anteo.

» Mastrángelo, M. (2009). “Oralidad y cultura radicalizada en el interior de la provincia de Córdoba, Argentina. Los casos de San Francisco y Río Cuarto en las décadas de 1930 y 1940". Antítesis, 2(3), 397-418.

» Mastrángelo, M. (2014). ““Rojos” y la cultura obrera argentina. Una aplicación de Raymond Williams a la historia oral”. Revista História \& Perspectivas, 27(51).

»Mastrángelo, M. (2011). Rojos en la Córdoba obrera. Buenos Aires: Imago Mundi. 
» Murmis, M. y Portantiero, J. C. ([1971] 2004). Estudios sobre los orígenes del peronismo, Buenos Aires: Siglo XXI.

»Pisani, A. (2016). "La clase obrera azucarera tucumana. Aproximaciones teórico-metodológicas para el estudio de su relación con el PRT-ERP entre 1966 y 1975". Historia Voces y Memoria, 9, 11-27.

"Pozzi, P. (2009). “Historia Social, historia militante: 'un producto colectivo'”. Revista História \& Perspectivas, 1(40).

"Pozzi, P. (2011). ““Era como una droga escucharlo a Perón”. Recordando la militancia treinta años más tarde (1955 a 1976)". CLIO: Revista de Pesquisa Históri$c a, 28.1$.

» Pozzi, P. (2015) “Trabajadores y procesos de politización y rebelión”. Esboços-Revista do Programa de Pós-Graduação em História da UFSC, 22(33).

»Rapaport, Mario (2012). Historia económica, política y social de la Argentina (1880-2000). Buenos Aires: Macchi.

» Thompson, E.P. (1989). Tradición, revuelta y conciencia de clase. Barcelona: Crítica.

»Torre, J. C. (1990). “Acerca de los estudios sobre la historia de los trabajadores en la Argentina". Anuario del IEHS, V, 209-220.

»Torre, J. C. (2011 [1990]). La vieja guardia sindical y Perón. Buenos Aires: Ediciones R y R.

»Varone, D. (1989). La memoria obrera. Buenos Aires: Cartago.

"Williams, R. (1965). The Long Revolution. Harmondsworth: Penguin.

»Williams, R. (2001). “La cultura es algo ordinario”, en John Higgins (Ed.). The Raymond Williams Reader. Oxford: Blackwell Publishers. 
